\title{
Across the great divide: Proteomics becoming an essential tool for animal and veterinary sciences
}

Author links open overlay panellngridMilleraAndré M.de AlmeidabP. DavidEckersallc

Show more

Add to Mendeley

Share

Cite

https://doi.org/10.1016/j.jprot.2021.104225Get rights and content

\section{Graphical abstract}

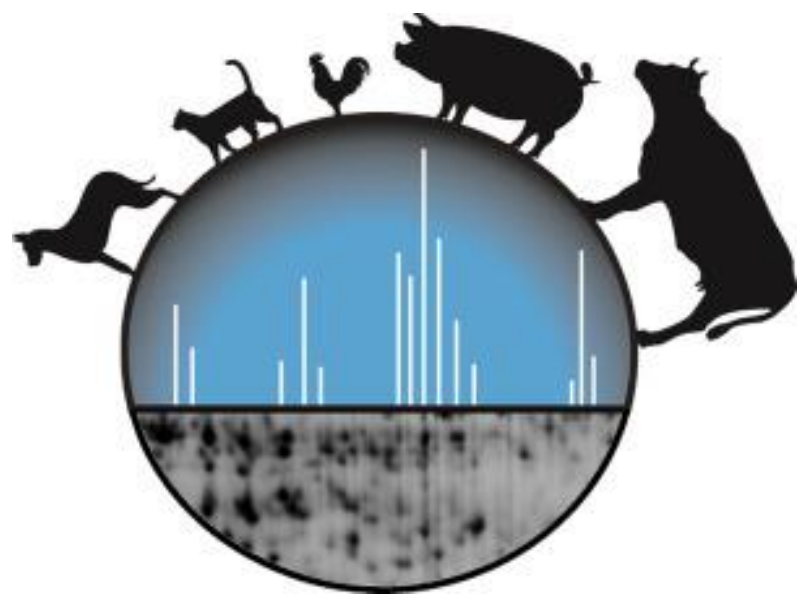

1. Download : Download high-res image (64KB)

2. Download : Download full-size image

- Previous article in issue

- Next article in issue

Since 2012, when the first and latest Thematic Issue of the Journal of Proteomics devoted to animal proteomics was published [1], there have been significant advances in the area, both in the extent of the applications and also in the use of more advanced technologies to this niche area of proteomic science. Although still not used as widely as in other areas of life sciences, such as biomedicine, the benefits of proteomics to extending the boundaries of knowledge in animal and veterinary science is being widely recognised. This has occurred to such an extent that an up-to-date Thematic Issue is fully justified and, as its predecessor, it should be influential in stimulating 
further interest and use by animal science researchers in the use of this particular technology. Indeed, it would be of great value if this issue could simultaneously encourage experts in animal and veterinary sciences to overcome any hesitation in seeking to apply advantages of proteomic analysis in their research and not only in research on farm animals but also in investigations of companion animals in health and disease. Furthermore, we hope to encourage those with extensive expertise in proteomics to recognise there is a considerable value and reward in applying their hard won expertise in alternative areas of comparative proteomics where surprising and illuminating findings await their endeavours. Accompanying the Thematic Issue is a position paper that examines the development of proteomics as applied to animal sciences beyond the boundary imposed by focussing on laboratory animal studies [2]. Herein, we introduce the papers included in the Thematic Issue in relation to their context in animal science and their implication for the future development of the field. The papers in the Issue describe the application of proteomics not only to terrestrial farm animals but also to fish in aquaculture and to companion animals. Species covered are cattle, sheep, goats, pigs, chicken, fish, dogs, cats and horses and topics include a wide range of physiological and pathophysiological phenomena, from reproduction to host responses to infection and nutritional studies.

Farm animal breeding is nowadays often based on assisted reproductive technologies (ART). Proteomics is able to assist, for instance by increasing the knowledge about the composition and physiology of female reproductive fluids [3] . Metabolomics data from plasma analysis may additionally provide valuable information about successful transfer of in vitro produced embryos [4]. Ruminant production is highly influenced by climate and seasonal changes, especially in tropical and sub-tropical regions. Increased knowledge about animal physiology of different breeds under these conditions gained by -omics is supposed to contribute not only to animal health, but also to their productivity and to higher quality of meat and dairy products [5]. Supplementation of animal feed by particular amino acids influences the proteome and metabolome of both the skeletal muscles and the blood of pre-weaned calves, for instance the level of antioxidant proteins []. Protein changes in beef can be traced back to animal stress [7], and protein biomarkers for beef tenderness have meanwhile be found [8]. Mammary gland secretions in the dry period and milk production have been studied over time [9] as well as the influence of Staphylococcus aureus infection 
on the milk proteome and peptidome of cows and goats $[\underline{10}, \underline{11]}$. Another product of small ruminants, wool of sheep, showed distinct proteome and fibre characteristics in a comparison of three particular genetic breeds from Portugal [12].

The manuscripts included in this issue dealing with pigs show an even larger variety in topics: They span from the proteome changes induced by exposure to the mycoestrogen zearalenone in the pig intestine [13] to a targeted proteome study on serum amyloid A isoforms in infection [14] and investigations of several brain areas in pigs raised under environmental enrichment [15]. Primary immune cells from transgenic pigs with a stable diabetic phenotype allowed the study of an early stage of diabetes mellitus [16]. Less known is about chicken leukocytes and therefore an approach was undertaken to better characterize a commonly used monoclonal antibody and to determine its true target [17]. Like in cattle, also in fish feed supplementation with amino acids, this time tryptophan, had an impact on physiology, metabolism and the proteome [18]. In addition, alteration in diet composition (linseed oil enrichment) caused changes in the liver phosphoproteome of a carnivorous marine fish [19].

Compared to other animal species, there is less in-depth information about protein components in equine body fluids, although different diseases would call for it [20]. Information is also lacking in equine sperm cryopreservation [21], the role of granulocytes in an autoimmune disease (equine recurrent uveitis) [22] and protein changes in chondrocytes during osteochondrosis [23]. In all those case, LC-MS based proteomics helped to collect more data on altered protein profiles.

Not all body fluids of dogs are equally well studied, as compiled for the less investigated ones in [24]. Among them is cerebrospinal fluid, whose protein composition was the topic of interest when comparing three different types/treatments of epilepsy [25]. Like in humans, also in the dog proteome and metabolome profiling of urine is a good means to diagnose chronic kidney disease [26]. Both, pyometra [27] and chronic valve disease [28] leave their traces in canine serum, the first mainly in acute phase protein levels, while not all detected changes in the latter disease were similar to those known in human pathophysiology. Together with protein from coagulation cascades, inflammation markers were also the main altered serum components in a study of feline congestive heart failure due to primary cardiomyopathy [29]. 
This Thematic Issue captures an essence of the expanding scope of research being performed in animal sciences, making use of the additional insights that are forthcoming from the use of current proteomic technology. This process should expand with the growing experience of a new generation of animal scientists who are familiar with the potential and technicalities of implementation. In combination with metabolomics which is also expanding in uses in animal sciences and the more established genomic and transcriptomic technologies, animal and veterinary sciences research will be able to take a truly systems biology approach to further explore fundamental and applied avenues of research, making significant contributions to the society.

\section{Acknowledgement}

We would like to thank Helena Ariño-Bassols (www.bioillustra.com) for designing the graphical abstact and the cover of this Thematic Issue.

\section{References}

P.D. Eckersall, A.M. Almeida, I. Miller

Proteomics, a new tool for farm animal science

J. Proteome, 75 (2012), pp. 4187-4189, 10.1016/j.jprot.2012.05.014

ArticleDownload PDFView Record in ScopusGoogle Scholar

[2]

A.M. Almeida, S.A. Ali, F. Ceciliani, P.D. Eckersall, L.E. Hernandez-

Castellano, R. Han, J.J. Hodnik, S. Jaswal, J.D. Lippolis, M. McLaughlin, I. Miller, A.K. Mohanty, V. Mrljak, J.E. Nally, P. Nanni, J.E. Plowman, M.D. Poleti, D.M. Ribeiro, P. R odrigues, B. Roschitzki, R. Schlapbach, J. Starič, Y. Yang, M. Zachut

Domestic Animal Proteomics in the 21st Century: A Global Retrospective and Viewpoint Analysis

J. Proteome, 241 (2021), p. 104220, 10.1016/j.jprot.2021.104220

ArticleDownload PDFView Record in ScopusGoogle Scholar

[3]

C. Itze-Mayrhofer, G. Brem

Quantitative proteomic strategies to study reproduction in farm animals: female reproductive fluids

J. Proteome, 225 (2020), p. 103884, 10.1016/j.jprot.2020.103884

ArticleDownload PDFView Record in ScopusGoogle Scholar 
E. Gómez, M. Muñoz, J. Gatien, S. Carrocera, D. Martín-González, P. Salvetti

Metabolomic identification of pregnancy-specific biomarkers in blood plasma of Bos taurus beef cattler after transfer of in vitro produced embryos

J. Proteome, 225 (2020), p. 103883, 10.1016/j.jprot.2020.103883

ArticleDownload PDFView Record in ScopusGoogle Scholar

\section{[5]}

D.M. Ribeiro, A. Salama, A.C.M. Vitor, A. Arguello, C.T. Moncau, E.M. Santos, G. Caja , J.S. de Oliveira, J.C.C. Balieiro, L.E. Hernández-

Castellano, M. Zachut, M.D. Poleti, N. Castro, S.P. Alves, A.M. Almeida

The application of omics in ruminant production: a review in the tropical and subtropical animal production context

J. Proteome, 227 (2020), p. 103905, 10.1016/j.jprot.2020.103905

ArticleDownload PDFView Record in ScopusGoogle Scholar

[6]

K. Yu, M. Matzapetakis, A. Horvatić, M. Terré, A. Bach, J. Kuleš, N. Yeste, N. Gómez, L. Arroyo, E. Rodríguez-Tomàs, R. Peña, N. Guillemin, A.M. de

Almeida, P.D. Eckersall, A. Bassols

Metabolome and proteome changes in skeletal muscle and blood of pre-weaning calves fed leucine and threonine supplemented diets

J. Proteome, 216 (2020), p. 103677, 10.1016/j.jprot.2020.103677

ArticleDownload PDFView Record in ScopusGoogle Scholar

$[7]$

F. Díaz, A. Díaz-Luis, V. Sierra, Y. Diñeiro, P. González, S. García-

Torres, D. Tejerina, M.P. Romero-Fernández, M. Cabeza de Vaca, A. Coto-

Montes, M. Oliván

What functional proteomic and biochemical analysis tell us about animal stress in beef?

J. Proteome, 218 (2020), p. 103722, 10.1016/j.jprot.2020.103722

ArticleDownload PDFView Record in ScopusGoogle Scholar

$[8]$

S. Boudon, D. Ounaissi, D. Viala, V. Monteils, B. Picard, I. Cassar-Malek

Label free shotgun proteomics for the identification of protein biomarkers for beef tenderness in muscle and plasma of heifers

J. Proteome, 217 (2020), p. 103685, 10.1016/j.jprot.2020.103685

ArticleDownload PDFView Record in ScopusGoogle Scholar

T.A. Reinhardt, J.D. Lippolis

[9]

Characterization of bovine mammary gland dry secretions and their proteome from the end of lactation through day 21 of the dry period

J. Proteome, 223 (2020), p. 103831, 10.1016/j.jprot.2020.103831 


\title{
$\underline{\text { ArticleDownload PDFView Record in ScopusGoogle Scholar }}$
}

\author{
[10]
}

M.F. Addis, E.M. Maffioli, F. Ceciliani, G. Tedeschi, V. Zamarian, F. Tangorra, M. Albe rtini, R. Piccinini, V. Bronzo

Influence of subclinical mastitis and intramammary infection by coagulase-negative staphylococci on the cow milk peptidome J. Proteome, 226 (2020), p. 103885, 10.1016/j.jprot.2020.103885

ArticleDownload PDFView Record in ScopusGoogle Scholar

S. Pisanu, C. Cacciotto, D. Pagnozzi, S. Uzzau, C. Pollera, M. Penati, V. Valerio Bronzo, M.F. Addis

Impact of Staphylococcus aureus infection on the late lactation goat milk proteome: new perspectives for monitoring and understanding mastitis in dairy goats

J. Proteome, 221 (2020), p. 103763, 10.1016/j.jprot.2020.103763

ArticleDownload PDFView Record in ScopusGoogle Scholar

J. Plowman, D. Harland, A. Campos, S. Silva, A. Thomas, J. Vernon, C. van

Koten, C. Hefer, S. Clerens, A. Almeida

The wool proteome and fibre characteristics of three distinct genetic ovine breeds from Portugal

J. Proteome, 225 (2020), p. 103853, 10.1016/j.jprot.2020.103853

ArticleDownload PDFView Record in ScopusGoogle Scholar

L. Soler, A. Stella, J. Seva, F.J. Pallarés, T. Lahjouji, O. Burlet-Schiltz, I.P. Oswald

Proteome changes induced by a short, non-cytotoxic exposure to the mycoestrogen zearalenone in the pig intestine

J. Proteome, 224 (2020), p. 103842, 10.1016/j.jprot.2020.103842

ArticleDownload PDFView Record in ScopusGoogle Scholar

A. Barslund Leuchsenring, C. Karlsson, L. Bundgaard, J. Malmström, P.M.H. Heegaard

Targeted mass spectrometry for Serum Amyloid A (SAA) isoform profiling in sequential blood samples from experimentally Staphylococcus aureus infected pigs J. Proteome, 227 (2020), p. 103904, 10.1016/j.jprot.2020.103904

-View PDF

Google Scholar 
L. Arroyo, D. Valent, R. Carreras, R. Pato, J. Sabrià, A. Velarde, A. Bassols

Neurobiology of environmental enrichment in pigs: changes in monoaminergic neurotransmitters in several brain areas and in the hippocampal proteome

J. Proteome, 229 (2020), p. 103943, 10.1016/j.jprot.2020.103943

ArticleDownload PDFView Record in ScopusGoogle Scholar

M. Weigand, R.L. Degroote, B. Amann, S. Renner, E. Wolf, S.M. Hauck, C.A. Deeg

Proteome profile of neutrophils from a transgenic diabetic pig model shows distinct changes

J. Proteome, 224 (2020), p. 103843, 10.1016/j.jprot.2020.103843

ArticleDownload PDFView Record in ScopusGoogle Scholar

C.A. Deeg, R.L. Degroote, M. Weigand, S. Hirmer, B. Amann, I.M. Giese, C. Wiedeman n, S.M. Hauck

CD11d is a novel antigen on chicken leukocytes

J. Proteome, 225 (2020), p. 103876, 10.1016/j.jprot.2020.103876

ArticleDownload PDFView Record in ScopusGoogle Scholar

M. Cerqueira, D. Schrama, T.S. Silva, R. Colen, S.A.D. Engrola, L.E.C. Conceição, P.M.

L. Rodrigues, A.P. Farinha

How tryptophan levels in plant-based aquafeeds affect fish physiology, metabolism and proteome

J. Proteome, 221 (2020), p. 103782, 10.1016/j.jprot.2020.103782

ArticleDownload PDFView Record in ScopusGoogle Scholar

G. Qin, D. Xu, B. Lou, R. Chen, L. Wang, P. Tan

iTRAQ-based quantitative phosphoproteomics provides insights into the metabolic and physiological responses of a carnivorous marine fish (Nibea albiflora) fed a linseed oil-rich diet

J. Proteome, 228 (2020), p. 103917, 10.1016/j.jprot.2020.103917 
E. Chiaradia, I. Miller

In slow pace towards the proteome of equine body fluids J. Proteome, 225 (2020), p. 103880, 10.1016/j.jprot.2020.103880 ArticleDownload PDFView Record in ScopusGoogle Scholar

F.E. Martín-Cano, G. Gaitskell Phillips, J.M. Ortiz-Rodríguez, A. SilvaRodríguez, A. Román, P. Rojo-Domínguez, E. AlonsoRodríguez, J.A. Tapia, M.C. Gil, C. Ortega-Ferrusola, F.J. Peña

Proteomic profiling of stallion spermatozoa suggests changes in sperm metabolism and compromised redox regulation after cryopreservation J. Proteome, 221 (2020), p. 103765, 10.1016/j.jprot.2020.103765 ArticleDownload PDFView Record in ScopusGoogle Scholar

M. Weigand, S.M. Hauck, C.A. Deeg, R.L. Degroote

Deviant proteome profile of equine granulocytes associates to latent activation status in organ specific autoimmune disease J. Proteome, 230 (2021), p. 103989, 10.1016/j.jprot.2020.103989 ArticleDownload PDFView Record in ScopusGoogle Scholar

E. Chiaradia, M. Pepe, P. Sassi, R. Mohren, P.L. Orvietani, M. Paolantoni, A. Tognoloni, M. Eveque, N. Tombolesi, B. Cillero-Pastor

Comparative label-free proteomic analysis of equine osteochondrotic chondrocytes J. Proteome, 228 (2020), p. 103927, 10.1016/j.jprot.2020.103927

ArticleDownload PDFView Record in ScopusGoogle Scholar

I. Miller, S. Schlosser, L. Palazzolo, M.C. Veronesi, I. Eberini, E. Gianazza 
Some more about dogs: proteomics of neglected biological fluids

J. Proteome, 218 (2020), p. 103724, 10.1016/j.jprot.2020.103724

ArticleDownload PDFView Record in ScopusGoogle Scholar

R. Baka, D. Eckersall, A. Horvatic, A. Gelemanovic, V. Mrljak, M. McLaughlin, L.V. At hanasiou, N. Papaioannou, I. Stylianaki, H.Q. Hanh, C.C. Chadwick, Z. Polizopoulou

Quantitative proteomics of cerebrospinal fluid using tandem mass tags in dogs with recurrent epileptic seizures

J. Proteome, 231 (2021), p. 103997, 10.1016/j.jprot.2020.103997

ArticleDownload PDFView Record in ScopusGoogle Scholar

E. Ferlizza, G. Isani, F. Dondi, G. Andreani, K. Vasylyeva, E. Bellei, A. de Almeida, M. Matzapetakis

Urinary proteome and metabolome in dogs (Canis lupus familiaris): the effect of chronic kidney disease

J. Proteome, 222 (2020), p. 103795, 10.1016/j.jprot.2020.103795

ArticleDownload PDFView Record in ScopusGoogle Scholar

J. Kuleš, A. Horvatic, N. Guillemin, R.F. Ferreira, R. Mischke, V. Mrljak, C. Chadwick, P.D. Eckersall

The plasma proteome and the acute phase protein response in canine pyometra J. Proteome, 223 (2020), p. 103817, 10.1016/j.jprot.2020.103817

ArticleDownload PDFView Record in ScopusGoogle Scholar

J. Kuleš, P. Bilić, A. Horvatić, A. Kovačević, N. Guillemin, B. Beer

Ljubić, A. Galan, I. Jović, M. Torti, I. Rubić, P.D. Eckersall, V. Mrljak

Serum proteome profiling in canine chronic valve disease using a TMT-based quantitative proteomics approach

J. Proteome, 223 (2020), p. 103825, 10.1016/j.jprot.2020.103825

ArticleDownload PDFView Record in ScopusGoogle Scholar 
M. Liu, P.D. Eckersall, V. Mrljak, A. Horvatić, N. Guillemin, A. Galan, L. Koster, A. Fre nch

\section{Novel biomarkers in cats with congestive heart failure due to primary cardiomyopathy}

J. Proteome, 226 (2020), p. 103896, 10.1016/j.jprot.2020.103896

ArticleDownload PDFView Record in ScopusGoogle Scholar

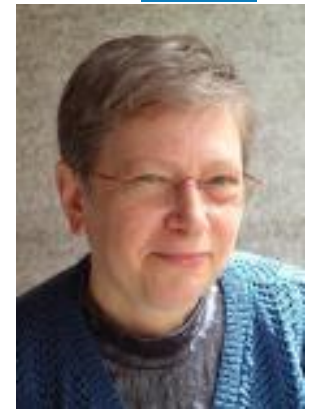

Ingrid Miller is at the Institute for Medical Biochemistry, Department for Biomedical Sciences, University of Veterinary Medicine Vienna, Austria. She holds a PhD in Environmental Technology awarded by the Wageningen University, the Netherlands. Originally trained in technical chemistry, she specialized early in protein chemistry and protein analytics, mainly on proteins of animal origin and from biological sources. Since more than three decades, her major focus is on proteomics and two-dimensional electrophoresis, with a special interest in methodology. She is a member of the editorial boards of several proteomic and analytical journals and a regular reviewer. She has been the Austrian representative in the EuPA General Council for several years, and was one of the founding members and vice president of the Austrian Proteomics Association. Since September 2009 she is Executive Editor for Animal Proteomics in the Journal of Proteomics. She is (co)author of over 120 peer-reviewed scientific papers, 10 book chapters, co-editor on a book about farm animal proteomics and one of the guest editors of four previous thematic issues in Journal of Proteomics (Farm Animal Proteomics, 2012; Environment and (Prote)-OMICS, 2016; SexDependency of the Proteome I and II, 2018 and 2019).

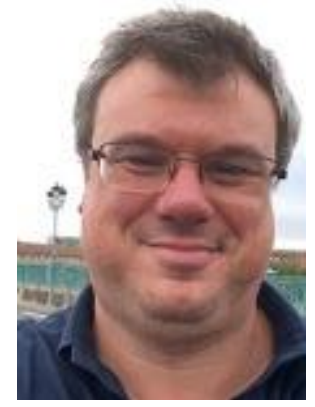

André M. de Almeida (Lisbon, 1974) is an Animal Scientist (1998) with a PhD in Biology (2005). He was a Researcher at the Tropical Research Institute (Lisbon, Portugal) from 2008-2014 and 
Associate Professor of Animal Nutrition and Animal Science at Ross University School of Veterinary Medicine (West Indies) from 2015-2016. In 2017, he joined the Faculty of Agriculture of the University of Lisbon (Portugal) where he teaches animal science and nutrition. He did research stays in Spain, South Africa, Australia and New Zealand. Research interests are mainly dedicated to studies of nutritional physiology and production of farm animals, particularly using Proteomics and Mass Spectrometry a research tool. He was Vice-Chair of COST action FA1002 - Proteomics in Farm Animals. In 2019, he received the award for the best scientific presentation at the Mediterranean Federation for Ruminant Health and Production annual meeting with a work on mammary gland Omics in dairy goats. He published 4 books, 24 international book chapters and over 100 peer-reviewed papers in ISI journals. He is presently editor-in-chief of Tropical Animal Health and Production, invited editor for the Journal of Proteomics and member of the board for Dairy and the Journal of Dairy Research. He is a regular reviewer for several journals in topics related to animal production and physiology and farm animal proteomics. He supervises five PhD students and different MSc students. He was a committee member of PhD thesis in Portugal, France, Spain and Italy.

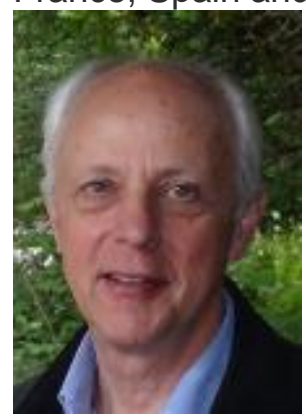

David Eckersall graduated from the University of Liverpool with a BSc in Biochemistry (1973) and a PhD in Biochemistry from University of Edinburgh (1977). Prof Eckersall is the Professor of Veterinary Biochemistry at the School of Veterinary Medicine, University of Glasgow. His research has been focused on the diagnostic applications of protein analysis and proteomics in veterinary medicine and has published over 200 peer reviewed papers, holds 4 patents and co-edited the first and second books on animal proteomics (Methods in Animal Proteomics, 2011, Wiley, Proteomics in Domestic Animals: from Farm to Systems Biology, 2019, Springer). He was the Chair of the COST Action for Farm Animal Proteomics (2011-14). He was awarded the Heiner Sommer Prize of the International Society for Animal Clinical Pathology for Lifetime Contribution to Animal Clinical Biochemistry in 2008, the Siemens Prize of the Division of Animal Clinical Chemistry of the American Association of Clinical Chemistry for Contributions to Animal Clinical Chemistry in 2010 and the Lifetime Achievements Award of the Comparative Clinical Pathology Association in 2016. In 2017 he took up a secondment to the University of Zagreb in Croatia to lead the Proteomics laboratory of the EU Funded VetMedZg project for improving Veterinary Molecular Medicine in Croatia. He is a Fellow of the Royal College of Pathologists, a Fellow of the Royal Society of Biology and a Member of the Academia Europaea. 